\title{
Salmonella host-pathogen interactions: a special topic
}

\author{
John S. Gunn* \\ Department of Microbial Infection and Immunity, Center for Microbial Interface Biology, The Ohio State University, Columbus, OH, USA \\ *Correspondence: gunn.43@osu.edu
}

Salmonellae are Enterobacteriaceae that cause a spectrum of diseases in humans and animals, including enteric (typhoid) fever and gastroenteritis (Coburn et al., 2007; Andrews-Polymenis et al., 2010). Most human Salmonella infections result in gastroenteritis and are caused by Salmonella enterica serovar Typhimurium (S. Typhimurium) or Salmonella enterica serovar Enteritidis acquired from contaminated food. Non-typhoidal Salmonella serotypes, such as $S$. Typhimurium, have a broad host range and infect a variety of animals. Interestingly, while these serotypes cause gastroenteritis in humans, they cause a systemic illness similar to typhoid fever in the mouse and cow (Andrews-Polymenis et al., 2010). In contrast, S. Typhi, the etiologic agent of typhoid (enteric) fever, infects only humans. Typhoid fever is a human systemic disease that is responsible for an estimated 21 million new infections annually resulting in approximately 600,000 deaths worldwide (Crump et al., 2004). It is an important health problem in developing countries and poses a significant risk to travelers. This illness has a mortality rate of $2-3 \%$ even with adequate antibiotic therapy, as antibiotic resistance of $S$. Typhi is a significant problem (Bhutta et al., 1991). Since the host range of $S$. Typhi is restricted to humans, the murine model system ( $S$. Typhimurium infection of susceptible inbred mice) has been widely used to study typhoid fever pathogenesis and immunity in the laboratory. Examining the progression of naturally acquired infection, the bacterium passes through the stomach and into the small intestine, preferentially invading, as do many other intestinal pathogens, through M-cells located in Peyer's patches in the distal ileum. After passage through the epithelium, the bacterium encounters, infects, replicates within, and disseminates (for systemic infections) primarily within macrophages. In this special topic, we explore the details of this infectious process through comprehensive reviews and summaries of key, recent publications.
One of the first contacts Salmonellae make in the intestine, particularly the colon, is with other bacteria or normal flora. Ahmer and Gunn (2011) describe the factors involved in overcoming "colonization resistance," mediated by normal flora and the gut innate immunity. In addition to mechanisms of innate immune defense subversion, they discuss various models and techniques that have been utilized to examine the S. Typhimurium-intestinal microbiota interplay. A key concept brought forth is that $S$. Typhimurium has been shown to be unable to colonize the mouse intestine in the absence of inflammation, as the normal flora in the non-inflamed state is able to effectively outcompete an avirulent (lacking inflammatory capacity) Salmonella intruder.

In addition to the normal flora, Salmonella must overcome numerous other gut barriers to infection, and must utilize its own weapons to enter through the non-phagocytic intestinal epithelial layer. Hallstrom and McCormick (2011) describe how Salmonella overcomes these hurdles and exploits the host defenses for their own purposes. They walk the reader through intestinal entry, the mucus layer, the intestinal epithelium [with focused discussion of M-cells as well as a paracellular pathway of entry (area between adjacent epithelial cells; tight junctions)], and into phagocytes where they are trafficked to the mesenteric lymph nodes.

Type III secretion systems are key to the virulence of several Gram-negative and enteric pathogens. The accepted dogma is that proteins are secreted by these systems directly from the cytoplasm of the bacterium to the cytosol of the host cell. However, the review of a manuscript by Akopyan et al. (2011) by Perrett and Zhou (2011) discusses data showing that this process may also occur by two steps - secretion to the bacterial surface followed by Type III secretion systems transport.

A manuscript by Knodler et al. (2010) reviewed here by Winter and Baumler (2011) describe the fate of some $S$. Typhimurium that find themselves within intestinal epithelial cells. In some instances, bacteria were surprisingly shown to escape the vacuole, replicate in the cytoplasm, and induce pyroptosis, which caused selective extrusion of these cells from the monolayer. This impacts both host clearance mechanisms and bacterial dissemination. Key to this inflammatory event is the activation of caspase-1, a topic that is reviewed by Miao and Rajan (2011). Upon bacterial sensing via membrane-bound toll-like receptors (TLRs) and intracellular NOD-like receptors (NLRs), pro-inflammatory pathways are activated which aid in clearance. Two of the NLR-containing complexes, NLRC4 and NLRP3 inflammasomes, and the activation of caspase-1 by these complexes, are key to this process, but Salmonella has also evolved mechanisms to subvert this response.

Increased details of Salmonella SPI-1 and SPI-2 function and how the factors that are secreted by these systems aid Salmonella in developing an intracellular niche are discussed by Malik-Kale et al. (2011). While the effectors for each pathogen are unique, the SPI-1 and SPI-2 type III secretion system translocated factors truly establish the intracellular niche for Salmonella, and their further understanding is key to a complete comprehension of host-pathogen interactions. They focus on the development of the unique Salmonella-containing vacuole and the role Salmonella plays in shaping its intracellular environment.

After progression of the bacterium through the epithelial barrier, Salmonellae encounter professional phagocytes. In the gut as well as following uptake/phagocytosis, Salmonellae are exposed to reactive oxygen (ROS) and nitrogen (RNS) intermediates. Henard and Vazquez-Torres (2011) describe the molecular targets of RNS (e.g., nitric oxide) as well as the ability of the bacterium to respond to this environment with mechanisms to both detoxify ROS and to repair damage- one of the important mechanisms 
of intracellular survival. They also discuss the interesting finding that Salmonella can exploit ROS to enhance virulence.

The review from Guiney and Fierer (2011) discusses SpvR, SpvB, and SpvC a positive transcriptional regulator and two SPI-2 secreted proteins, respectively. Interestingly, these loci are not found in S. Typhi but are strongly associated with non-typhoidal bacteremia causing strains. Though unclear concerning their role in virulence, they are an example of the species-specific virulence traits common in the Salmonellae.

All of the pathogenic events discussed in this Salmonella special topic rely upon effective environmental sensing and gene regulation. Salmonella possesses a complex regulatory network that has been studied in pieces, but not in toto where these regulatory network interconnections would be defined. Using high throughput genomic and proteomic assays and newly developed computational approaches for data integration, McDermott et al. (2011) show how these complex networks and inter-system regulatory pathways are being defined on a genome wide scale. This allows for a never before seen "30,000 foot view" of the interworkings of the virulence regulatory network in Salmonella.

\section{ACKNOWLEDGMENTS}

This work was supported by The National Institutes of Health grants AI066208 and AI043521.

\section{REFERENCES}

Ahmer, B. M., and Gunn, J. S. (2011). Interaction of Salmonella spp. with the intestinal microbiota. Front. Microbiol. 2:101. doi: 10.3389/fmicb.2011.00101

Akopyan, K., Edgren, T., Wang-Edgren, H., Rosqvist, R., Fahlgren, A., Wolf-Watz, H., and Fallman, M. (2011) Translocation of surface-localized effectors in type III secretion. Proc. Natl. Acad. Sci. U.S.A. 108, 1639-1644.

Andrews-Polymenis, H. L., Baumler, A. J., McCormick, B. A., and Fang, F. C. (2010). Taming the elephant: Salmonella biology, pathogenesis, and prevention. Infect. Immun. 78, 2356-2369.

Bhutta,Z.A., Naqvi, S. H., Razzaq, R. A., and Farooqui, B. J. (1991).Multidrug-resistant typhoid in children:presentation and clinical features. Rev. Infect. Dis. 13, 832-836.

Coburn, B., Grassl, G. A., and Finlay, B. B. (2007) Salmonella, the host and disease: a brief review. Immunol. Cell Biol. 85, 112-118.

Crump, J. A., Luby, S. P., and Mintz, E. D. (2004). The global burden of typhoid fever. Bull. World Health Organ. 82, 346-353.

Guiney, D. G., and Fierer, J. (2011). The role of the spv genes in Salmonella pathogenesis. Front. Microbiol. 2:129. doi: $10.3389 /$ fmicb.2011.00129

Hallstrom, K., and McCormick, B. A. (2011). Salmonella interaction with and passage through the intestinal mucosa: through the lens of the organism. Front. Microbiol. 2:88. doi: 10.3389/fmicb.2011.00088

Henard, C. A., and Vazquez-Torres, A. (2011). Nitric oxide and Salmonella pathogenesis. Front. Microbiol. 2:84. doi: 10.3389/fmicb.2011.00084
Knodler, L.A.,Vallance, B. A.,Celli, J., Winfree, S., Hansen, B., Montero, M., and Steele-Mortimer, O. (2010). Dissemination of invasive Salmonella via bacterialinduced extrusion of mucosal epithelia. Proc. Natl. Acad. Sci. U.S.A. 107, 17733-17738

Malik-Kale, P., Jolly, C. E., Lathrop, S., Winfree, S, Luterbach, C., and Steele-Mortimer, O. (2011). Salmonella - at home in the host cell. Front. Microbiol. 2:125. doi: 10.3389/fmicb.2011.00125

McDermott, J. E., Yoon, H., Nakayasu, E. S., Metz, T. O, Hyduke, D. R., Kidwai, A. S., Palsson, B. O., Adkins, J. N., and Heffron, F. (2011). Technologies and approaches to elucidate and model the virulence program of Salmonella. Front. Microbiol. 2:121. doi: 10.3389/ fmicb.2011.00121

Miao, E. A., and Rajan, J. V. (2011). Salmonella and caspase-1: a complex interplay of detection and evasion. Front. Microbiol. 2:85. doi: 10.3389/fmicb.2011.00085

Perrett, C. A., and Zhou, D. (2011). Type three secretion system effector translocation: one step or two? Front. Microbiol. 2:50. doi: 10.3389/fmicb.2011.00050

Winter, S. E., and Baumler, A. J. (2011). Salmonella exploits suicidal behavior of epithelial cells. Front. Microbiol. 2:48. doi: 10.3389/fmicb.2011.00048

Received: 29 August 2011; accepted: 29 August 2011; published online: 19 September 2011

Citation: Gunn JS (2011) Salmonella host-pathogen interactions: a special topic. Front. Microbio. 2:191. doi: 10.3389/ fmicb.2011.00191

This article was submitted to Frontiers in Cellular and Infection Microbiology, a specialty of Frontiers in Microbiology.

Copyright $\odot 2011$ Gunn. This is an open-access article subject to a non-exclusive license between the authors and Frontiers MediaSA, which permits use, distribution and reproduction in other forums, provided the original authors and source are credited and other Frontiers conditions are complied with. 